\title{
Convective het transfer along slender cylinders
}

\author{
by J-M. Buchlin and R. Tasse
}

von Karman Institute for Fluid Dynamics, Rhode-Saint-Genèse, Belgium

\begin{abstract}
An experimental investigation of the forced convection along vertical, slender cylinders aligned in a uniform air flow is performed using quantitative infrared thermography. In the laminar regime, the heat transfer data are correlated in terms of a local dimensionless curvature parameter and validate the thermal boundary layer model for a single slender cylinder. Turbulent heat transfer occurs when the standoff distance between two parallel, slender cylinders is sufficiently small to affect the axisymmetry of the boundary layer. An enhancement factor of three can be achieved at the transition region. In the turbulent regime, the curvature parameter alone does not describe the thermal exchange.
\end{abstract}

\section{NOMENCLATURE}

$\begin{array}{ll}a & \text { Cylinder radius } \\ B i & \text { Biot number } \\ d_{i} & \text { Stand-off distance } \\ F_{i j} & \text { View factor } \\ N u & \text { Nusselt number } \\ q & \text { Heat flux } \\ R e & \text { Reynolds number } \\ T & \text { Temperature } \\ U_{\theta} & \text { Air velocity } \\ x & \text { Axial postion } \\ X_{0} & \text { Location of the wire attachment }\end{array}$

\section{Greek symbols}

$\alpha \quad$ Angle of incidence

$\delta \quad$ Boundary layer thickness

e Emissivity coefficient

n Kinematic viscosity

$\sigma$ Stefan-Boltzmann constant

$\xi$ Curvature parameter

\section{Subscripts}

a Based on the radius

c Convection

e Ambient conditions

$r$ Radiation

$x$ Axial

w Wire

\section{Superscript \\ * Transition}

\section{Introduction}

Heat transfer by forced convection along slender cylinders is of great interest for the polymer-fibre spinning process where careful control of the yarn quenching has a strong bearing on the final product quality [1]. The thermal exchange between the wires and air has been intensively investigated through theoretical and numerical approaches [2-4]. Surprisingly, experimental studies of the axial thermal convection around a slender cylinder are rather sparse and generally show contradictory results. They lead to global correlations without a description of the thermal boundary layer [5-6] or they are addressed to cylinders with a large diameter and without curvature effect [7]. When carried out on industrial lines, they do not provide the relevant information needed to extract heat transfer data [8].

The present paper constitutes an additional contribution in illustrating the applicability of quantitative IR thermography to determine the convective heat-transfer distribution along vertical, slender cylinders simulated by long heated wires. It summarises some features of a thorough aero-thermal study carried out at the VKI on the cooling of synthetic fibres [9]. Only the cases of a fixed single vertical cylinder ( mono-wire) and a pair of two parallel cylinders (biwire) aligned with a uniform air flow are considered. After describing the experimental set-up and the procedure to collect and reduce the data, the paper emphasises the effect of the flow and geometrical parameters on the thermal exchange. Conclusions are drawn on the behaviour in the laminar and turbulent regimes. 


\section{Test Facility}

The test set-up is the VKI L10 wind tunnel sketched in figure 1a. The test section is a vertical $1.8 \mathrm{~m}$ long channel with a cross-section of $0.2 \times 0.2 \mathrm{~m}^{2}$. The inlet is connected to a settling chamber provided with fine screens and honeycomb to make the flow distribution uniform. The convergent section has a contraction ratio of $5.76: 1$. The freestream is produced by a fan driven by an asynchronous motor. The air velocity ranges from 1 to $12 \mathrm{~m} / \mathrm{s}$ and the turbulence level does not exceed $0.5 \%$. The slender cylinders are metallic wires $1.7 \mathrm{~mm}$ in diameter and are heated by the Joule effect. A detailed description of the wire attachment is given in [9]. In the experiments with a single slender cylinder, the heating wire is attached, via an aerodynamically-shaped piece in bakelite, to a nylon yarn. The latter is fixed in the settling chamber by means of a small ogive. The mounting system for the case of a pair of slender cylinders is shown in figure $1 b$. The stand-off distance $d_{i}$ and the parallelism of the two wires is insured by two devices in bakelite. The upstream piece is attached to an ogive by a nylon wire as in the mono-wire case; the downwind piece is plugged into a rotating cylindrical device that allows accurate adjustment of the wire position.

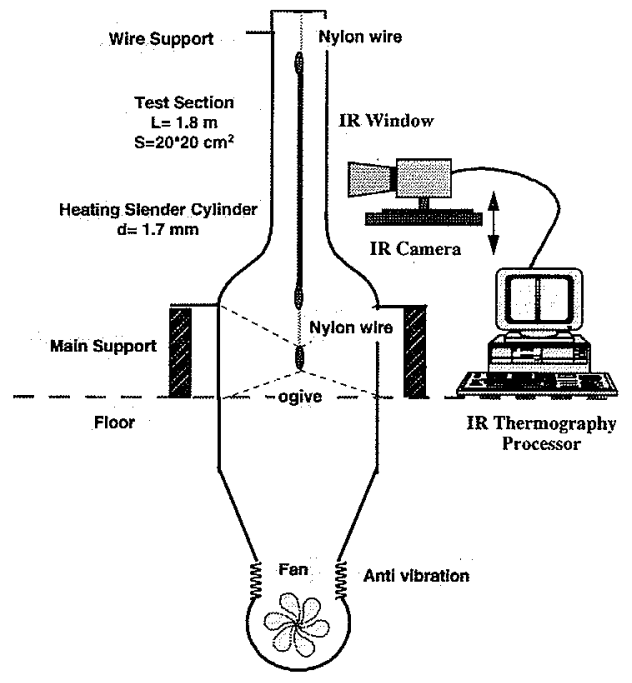

a)

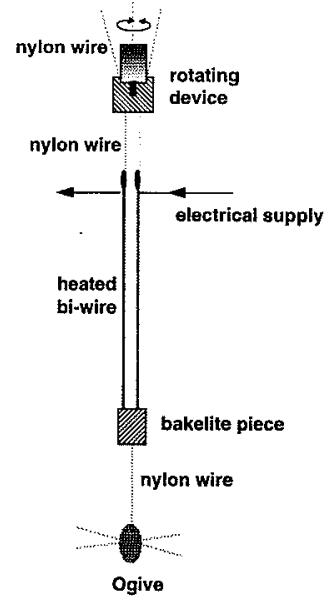

(b)

Fig. 1. (a) Schematic diagram of the test set-up; (b) Bi-wire mounting.

The walls of the test section are in Plexiglas. One is composed of several windows made of a special plastic film, transparent to near infrared radiation, for the application of quantitative IRthermography. The wires are coated with a black paint of emissivity $\varepsilon_{\mathrm{w}}=0.96$ to improve the thermography measurements and to allow correction for the heat loss by radiation. The IR scanner is the AGEMA Thermovision 900 system with an $\mathrm{HgCdTe}$ detector sensitive in the 8$12 \mu \mathrm{m}$ wavelength range and cooled by liquid nitrogen. The measurable temperature range is $30^{\circ} \mathrm{C}$ to $1500^{\circ} \mathrm{C}$ with a published thermal sensitivity of $0.1^{\circ} \mathrm{C}$. The camera is equipped with a standard optical set-up, $10^{\circ}$ vertical $\times 5^{\circ}$ horizontal, giving an instantaneous field of view (IFOV) of 0.76 mrad. An intermediate ring leads to a focal distance of $0.45 \mathrm{~m}$ and consequently improves the resolution of the camera, which is a major point when working with small cylinder diameters. The camera is mounted on a vertical displacement mechanism to scan the entire cylinder length of $1 \mathrm{~m}$. The rate of acquisition is 25 thermogrammes per second. The calibration of the camera is performed in conditions similar to the real measurements. The heating sources 
are hollow tubes of Monel alloy of $2 \mathrm{~mm}$ and $3 \mathrm{~mm}$ in external diameter respectively; they are also painted black and heated by the Joule effect. Two thermocouples are inserted in each tube filled with a thermally conducting gel $(55 \mathrm{~W} / \mathrm{m} . \mathrm{K})$. Such calibration devices are characterised by very small Biot number ( i.e. $B i_{a}<0.001$ ), so that the temperature measured by the thermocouples corresponds to the surface temperature of the model. The effect of the plastic film on the response of the IR camera is quantified during calibration.

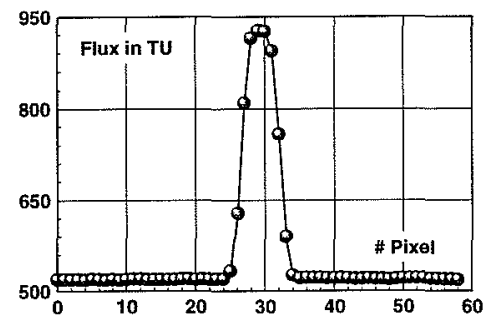

(a)

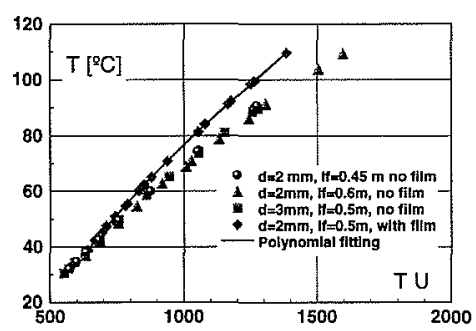

(b)

Fig. 2. (a) Transverse distribution of TU ; (b) Calibration curve.

The calibrating thermogramme is illustrated in figure $2 a$ where a typical transverse distribution of thermal units (TU) across the wire is plotted. The Gaussian profile results from the combined effect of the slit response function of the camera and the curvature of the slender cylinder. The maximum value, located on the generating line of the wire, is plotted versus the temperature measured by the thermocouple in figure $2 b$. Whatever the focal distance of the camera $l_{f}$ and the wire temperature $T_{w}$, the data fit the same curve, indicating that the limit of the resolution of the camera has not been yet reached. A similar outcome is expected in the wind tunnel conditions, since the view angle of $2.79 \mathrm{mrd}$ selected for the experiments, in within the range of 2.67 to 3.28 mrd covered by the calibration tests. Furthermore, the noticeable drop of the TU-level caused by the plastic film at high temperature, shown in figure $2 b$, confirms the need for a calibration procedure.

\section{Typical Results}

\subsection{Single slender cylinder}

In order to get rid of distortion induced by the vertical misalignment of the slender cylinder in the air flow, preliminary tests are conducted to identify the effect of the angle of incidence $\alpha$. The results are plotted in figure 3 . At any axial location $x$, the temperature exhibits a maximum value when the incidence is zero. The perfect symmetry of the temperature variation with $\alpha$ indicates that the radial thermal conduction is strong enough to mask any angular variation of the heat transfer coefficient due to incidence.

Temperature distributions along the slender cylinder are measured for air velocity ranging from 3 to $8 \mathrm{~m} / \mathrm{s}$ and heat flux varying from 1000 to $5000 \mathrm{~W} / \mathrm{m}^{2}$. The recorded time-variation of the local temperature confirms that steady state is reached after a few seconds. Typical results are shown in figure 4. The dispersion of the data is caused by the slight changes of the wire diameter [9].

The local convective heat-transfer coefficient $h(x)$ is determined by application of the Newton definition: 


$$
h(x)=\frac{q_{c}(x)}{T_{w}(x)-T_{e}}
$$

where $T_{\theta}$ is the air temperature of the freestream and $q_{c}$ is the heat flux removed by convection $=q_{J}-q_{r}(x)$. The heat loss by thermal radiation, $q_{r}(x)$, is derived from the radiosity concept assuming a cylindrical surface small compared to the surface of the surrounding walls at constant temperature $T_{\mathrm{e}}$ :

$$
q_{r}(x)=\varepsilon_{w} \sigma\left(T_{w}^{4}(x)-T_{e}^{4}\right)
$$

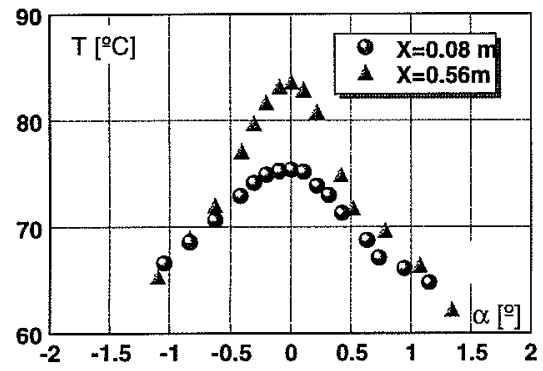

Fig. 3. Effect of the angle of incidence on the slender cylinder temperature.

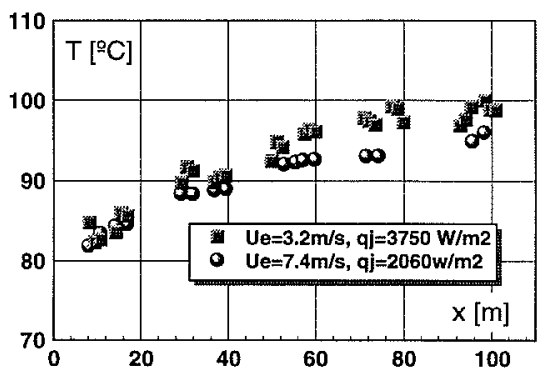

Fig. 4. Axial temperature distribution along the slender cylinder.

The $q_{r}$-value can be $20 \%$ of the Joule heating while corrections due to thermal conduction along the wire appear to be negligible except near the point where transition to turbulence occurs. The conventional dimensionless form of the Nusselt number based on the cylinder radius $a$ is adopted: $N u_{a}=h . a / k_{a}$; the uncertainty is $8 \%$.

The effect of the position of the upstream end of the wire on the thermal exchange is displayed in figure 5. The abscissa $X_{0}$ represents the location of the ogive of the wire mounting with respect to the inlet of the convergent section (a negative $X_{0}$-value corresponds to the upstream position). Identical results are obtained when the upstream end of the wire is located in the settling chamber $\left(X_{0}=-0.2\right.$ or $\left.0 \mathrm{~m}\right)$, a zone of low air velocity. In contrast, when the attachment device is moved into an accelerating flow area $\left(X_{0}=0.4 \mathrm{~m}\right)$ the presence of the bakelite obstacle induces turbulent flow and a subsequent increase in the heat transfer. Therefore, to avoid any flow distorsion, the configuration with $X_{0}=-0.2 \mathrm{~m}$ is maintained for the tests. So as to compare the IR-data with theoretical predictions, the local Nusselt number $N u_{a}$ is plotted in figure 6 versus the dimensionless co-ordinate $\xi$ that accounts for the curvature effect of the cylinder:

$\xi=\sqrt{\frac{x \cdot v}{U_{e} \cdot a^{2}}}=\frac{\sqrt{\operatorname{Re}_{x}}}{\operatorname{Re}_{a}}=\sqrt{\frac{x}{a} \cdot \frac{1}{\operatorname{Re}_{a}}}$

Note that in laminar flow $\xi$ is proportional to the ratio of the boundary-layer thickness to the wire radius $\delta / a$. The Nusselt number is a decreasing function of the parameter $\xi$ as predicted by the VKI laminar boundary-layer model [9]. The good agreement observed between the IR data and the theory supports the fact that the boundary layer is laminar up to the $\xi$-value of 3 . The following correlation is proposed: 


$$
N u_{a}=\frac{C_{o}}{\sqrt{\xi}} \quad \text { with } \mathrm{C}_{\circ}=0.85
$$

\subsection{Pair of slender cylinders}

In experiments conducted with a pair of slender cylinders, the correction for the radiation is more involved than in the case of the monowire. The radiative exchange is between the two cylinders and with the walls of the test section. The modelling leads to a linear algebraic system for the radiosity $J_{j}$ :

$$
\sum_{j=1}^{j=N}\left[\delta_{i j}-\left(1-\varepsilon_{i}\right) \cdot F_{i j}\right] J_{j}=\varepsilon_{i} \sigma T_{i}^{4}
$$

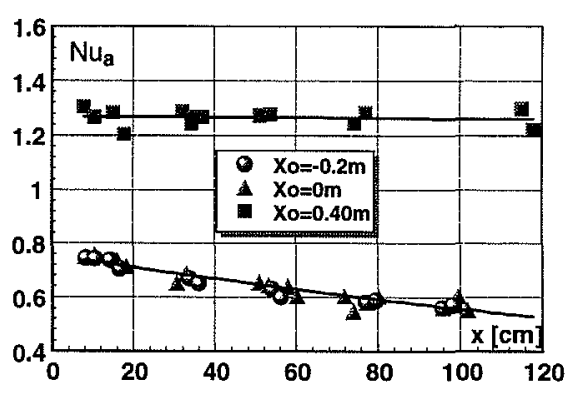

Fig. 5. Effect of the wire starting position on the heat-transfer coefficient

where $\delta_{i j}$ is the Kronecker delta function and $F_{i j}$ the view factor between the bodies $i$ and $j$. Within the acceptable approximation that the surface of the cylinders is small compared to that of the test section and the two cylinders have nearly the same temperature, the relation (5) leads to the following simplified expression for the radiative heat flux:

$$
q_{\mathrm{rad}}=(1-\varepsilon) \cdot F_{12} \sigma\left(T_{w}^{4}-T_{a}^{4}\right)
$$

The prediction by Eq. (5) and (6) differs by less than $3 \%$ when the temperature difference between the wires does not exceed $30^{\circ} \mathrm{C}$.

Tests are performed for two normalized standoff distances $d_{i} / a=7.6$ and 9.5 , respectively. Air velocity ranges from 2 to $7 \mathrm{~m} / \mathrm{s}$. The Nusselt number $\mathrm{Nu}_{a}$ obtained for $d_{i} / a=9.5$ is compared to the mono-wire results in figure 7. The values of $N u_{a}$ remain independent of the velocity, indicating that the the choice of $\xi$ as a representative dimensionless parameter is still valid for the two-wire configuration. The detailed LDV investigation of the boundary layer reported in [9] shows that, for such a stand-off

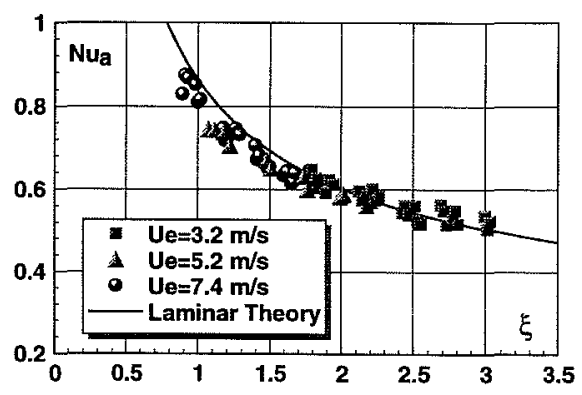

Fig. 6. Nusselt number versus the curvature parameter for a single slender cylinder.

distance, the interaction does not affect too much the axisymmetric character of the flow pattern around the wires but produces a mass flow deficit in the zone between the two cylinders. Therefore, it is consistent to observe a trend similar to the mono-wire case with a lower heattransfer coefficient. The deviation from the mono-wire data grows as $\xi$ increases, revealing a stronger boundary-layer interaction but without triggering transition to turbulence.

The results obtained for $d_{i} / a=7.6$ are plotted in figure 8 . They exhibit a different thermal behaviour. At a given axial position $x^{\star}$, the Nusselt number experiences a sharp increase that could be more than a factor of three for the largest air velocity. Such a jump corresponds to the transition from a laminar to a turbulent boundary layer. The location of $x^{*}$ moves upstream as the freestream velocity increases. Ahead of $x^{*}$ all the data fit the laminar curve and $\xi$ remains a relevant descriptive parameter. Here, the trend of the $d_{i} / a=7.6$-data is similar to that obtained with $d_{i} / a=9.5$ (see figure 7 ) except that the deviation from the mono-wire results is now larger due to a prominent boundary-layer interaction. Above $x^{*}$, in the turbulent regime, $\xi$ is not sufficient to correlate the heat-transfer coefficient. 


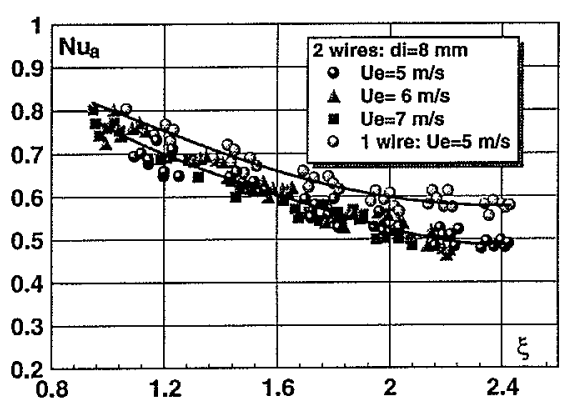

Fig. 7: Nusselt number for a pair of slender cylinders: $d_{i} / a=9.5$

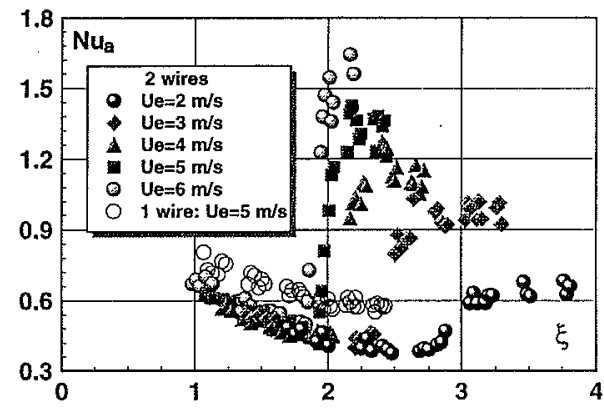

Fig. 8. Nusselt number for a pair of slender cylinders: $d_{i} / a=7.6$

\section{Conclusions}

Steady-state measurements of the heat transfer along vertical, slender cylinders aligned within a uniform air stream are obtained using quantitative infrared thermography. Thermogramme calibration and corrections for the radiative exchange are performed. As long as the boundary layer remains laminar, the local Nusselt number, based on the cylinder radius $\mathrm{Nu}_{a}$, can be correlated with the local curvature parameter $\xi$. In the case of a single slender cylinder, no turbulent heat transfer is observed for $\xi \leq 3$. The IR data validate a laminar boundary-layer model and a simple correlation is proposed. In the case of two parallel slender cylinders, the thermal exchange depends on the stand-off distance $d_{i}$. In the laminar regime, the interaction between the two boundary layers leads to a deficit of mass flow which results in a decrease of the heat transfer coefficient relative to the mono-cylinder case. For $d_{i} / a$-values smaller than 8 the occurrence of a turbulent boundary layer and enhancement of heat transfer by a factor three can be expected. In the turbulent regime, $\xi$ is not sufficient to correlate the heat-transfer data.

\section{Acknowledgements}

The authors gratefully thank Rhône-Poulenc, France for providing support in this research.

\section{REFERENCES}

[1] Ziabicki, A.: Fundamentals of fibre formation, John Wiley \& sons, 1976.

[2] Bourne, D.E. and Davies, D.R.: Heat transfer through boundary layer on a circular cylinder in axial incompressible flow. Quart. J. Mech. and Applied Math. Vol XI, Pt. 1, pp. 52-66, 1958.

[3] Sparrow, E.M. and Yu, H.S.: Local non-similarity thermal boundary layer solutions. Journal of Heat Transfer, pp. 328-334, 1971.

[4] Heckel, J.J.; Chen, T.S.; Armaly, B.F.: Natural convection along slender cylinders with variable surface heat flux. Int. JHMT, Vol 32, No 8, pp. 1431-1442, 1989

[5] Andrews, E.H.: Cooling of a spinning thread-line: Brit.J. of App. Phy. Vol 10, January 1959.

[6] Sano, Y. and Nishikawa: Kagaku Kogaku, Vol. 28, pp. 275, 1964

[7] Eckert, E.R.G.; Eichorn, R.; Eddy, T.L.: Measurements of temperature profiles in laminar and turbulent boundary layers on a cylinder with non-uniform wall temperature: ARL Technical note, University of Minnesota, December 1960.

[8] Chen, S.; Yu, W.; Spruiell, J.E.: On-line studies of structure development during melt spinning of Poly (butylene Terephthalate). Journal of Applied Polymer Science, Vol. 34, pp. 1477$1492,1987$.

[9] Tasse, R.: Etude théorique et expérimentale du refroidissement à l'air de fils synthétiques. Thèse de Doctorat en Sciences Appliquées, ULB-IVK, Septembre 1995. 